\title{
Outcome of PUV Patients Following Ablation in a Tertiary Teaching Hospital in Addis Ababa, Ethiopia
}

\author{
Hiwote G Assefa' \\ Hanna Getachew ${ }^{2}$ \\ Amezene Tadesse ${ }^{2}$ \\ Woubedel Kiflu ${ }^{2}$ \\ Fisseha Temesgen ${ }^{2}$ \\ Belachew Dejene ${ }^{2}$ \\ Tihitena Negussie Mammo ${ }^{2}$ \\ 'Department of Surgery, St Paul's \\ Hospital, Millennium Medical College, \\ Addis Ababa, Ethiopia; ${ }^{2}$ Department of \\ Surgery, Addis Ababa University School \\ of Medicine, Addis Ababa, Ethiopia
}

Correspondence: Hiwote G Assefa;

Tihitena Negussie Mammo

Email hiwotesc@gmail.com;

tihutin@yahoo.com
Background: Posterior urethral valve (PUV) is the most common cause of congenital lower urinary tract obstruction in boys. It is considered that early diagnosis and intervention have good outcomes in terms of renal function, though the varying extent of embryological insult requires these boys to remain in extended follow-up and care.

Objective: To assess the renal outcome of patients following PUV ablation.

Methods: This was a descriptive retrospective study. Data were collected from the operation logbooks of patients from 2015 to 2019 that had been admitted to the Tikur Anbessa Specialized Hospital pediatric surgery unit with a working diagnosis of PUV and had ablation done primarily or following diversion. Data were collected from January to April 2020 and analyzed using SPSS 25. $P$ value $\leq 0.05$ was considered significant.

Results: Seventy patients were analyzed and followed for 3 years for the development of postoperative chronic kidney disease (CKD) after PUV ablation. Postoperative CKD was found in $52.9 \%$ of patients and end-stage renal disease in $2.9 \%$. Risk factors associated with postoperative CKD were the presence of preoperative and postoperative proteinuria, postoperative hypertension, and elevated nadir serum creatinine. Results also showed that a delay between the development of vesicostomy and ablation had a significant correlation with renal outcome. Elevated nadir serum creatinine, postoperative proteinuria, and delay between the development of vesicostomy and ablation were found to be independent risk factors of development of CKD.

Conclusion: There was a high rate of CKD development in patients who had had ablation for PUV, which was comparable to other studies. Three variables were found to be independent risk factors for the progression of $\mathrm{CKD}$, unlike other findings seen in low- and middle-income countries. Keywords: posterior urethral valve, primary ablation, vesicostomy, nadir serum creatinine, proteinuria, chronic kidney disease, end-stage renal disease

\section{Introduction}

Posterior urethral valves (PUV) is the most common cause of bladder-outlet obstruction in male neonates and infants. ${ }^{1}$ This is a congenital obstruction of the posterior urethra believed to be caused by valve-like leaflets or membranes, where it is also called congenital obstruction of the posterior urethral membrane (COPUM). There are three types of PUV according to Young et al's classification. Type 1 is the most common type, occurring in $90 \%-95 \%$ of patients. $^{2}$

Congenital anomalies of the urinary tract affect up to one in every 500 pregnancies, and obstructive uropathy accounts for most of these cases. ${ }^{2,3}$ It is considered that PUV have an incidence of 1.6-2.1 per 10,000 live births. ${ }^{2}$ A study done at Tikur Anbessa Specialized Hospital (TASH) on 5 years' pediatric surgical 
admissions showed that congenital obstruction of the posterior urethral membrane was one of the ten most common congenital admissions, comprising $2.5 \%$ of all surgical admissions. ${ }^{4}$

With distal obstruction, there is increased voiding pressure that is compensated by hypertrophy and dilation of the bladder. The bladder dysfunction also leads to transmission of pressure to the ureter and renal pelvis, leading to urine-reflux, worsening of hydronephrosis and renal dysfunction. This valve-bladder syndrome is considered to have a vicious cycle. Eventually, the bladder decompensates with poor contractility and upper-tract dilation. ${ }^{5,6}$

Pressure transmission on the glomerular units result in architectural changes. The renal dysfunction seen in PUVs is considered to arise due to either obstructive uropathy, as mentioned above, or from renal dysplasia. ${ }^{5}$

Vesicoureteral reflux (VUR) occurs in $50 \%-70 \%$ of patients with PUV. The high-grade VUR that is sometimes seen in children, mostly occurs in the poorly functioning ipsilateral renal unit and the contralateral functioning unit is sparedwhich is a "pop off" mechanism also known as vesicoureteral reflux dysfunction (VURD) . This has been postulated to be a physiological mechanism that saves the contralateral kidney. However, long-term studies have shown that VUR dysfunction does not improve renal prognosis. $^{5,7}$

PUV is mostly diagnosed during antenatal follow-up and can be confirmed with postnatal imaging. ${ }^{5}$ Interventions can be done as early as possible, even during the intrauterine period, but embryological insult to the bladder and kidneys manifesting to a varying extent require these boys to remain in extended follow-up and care. ${ }^{5}$

After birth, these patients should undergo investigation to reach a diagnosis early so as to intervene with ablation or vesicostomy in the neonatal period. Earlier interventions are to lessen the degree of renal dysfunction. ${ }^{8}$

A study in Canada concluded that valve ablation is the main stay of treatment for patients with PUV. prenatal and postnatal factors, such as renal dysplasia and urinary tract infrection respectively, rather than the PUV treatment dictate the long term renal and radiological outcome. ${ }^{18}$

This study was performed to observe renal outcomes after ablation and to evaluate the effect of multiple risk factors on renal function. The study also aids in determining renal outcomes if intervention is done early. All these will lead to development of a guideline on how to diagnose and treat these patients early so as to improve renal outcomes.

\section{Methods}

This study was conducted on patients that had been treated at Tikur Anbessa Specalized Hospital (TASH) with PUV ablation. TASH is one of the biggest tertiary teaching hospitals in Addis Ababa, capital of Ethiopia. A descriptive retrospective cross-sectional study design was used. Data were collected from operation logbooks of patients that had been admitted from January 2015 to January 2019 to the TASH pediatric surgery unit with a working diagnosis of PUV, and ablation was done either primarily or following diversion. The sample size was calculated based on the admission rate of PUV patients to the hospital and was calculated to be 114 . We mainly focused on identifying those patients with progression of chronic kidney disease (CKD) following ablation or those that had newly developed CKD or end-stage renal disease (ESRD).

The major risk factors of progression to CKD were identified from textbooks and published papers and used as independent variables. Because of the adequate sample size, parametric statistical analysis was used. All independent variables were first analyzed for frequency, followed by crosstabulation. $P \leq 0.05$ was considered significant. Significant variables were then analyzed by bivariate analysis. Variables that were significant on that were analyzed by multivariate analysis, and those significant on that were considered independent risk factors of development and progression of CKD.

The ethics committee of the Addis Ababa University College of Health Science Department of Surgery approved the research, and since it was a retrospective study with chart review, no consent was taken from individual patients after a consent waiver QAS had been granted by the committee. All patients data were maintained with confidentiality, in accordance with the Declaration of Helsinki. A questionnaire was formulated and data collected from January to April 2020 and analyzed using SPSS 25. $P \leq 0.05$ was considered significant.

Operational definitions were set for both the dependent and independent variables:

\section{Dependent variables $1,2,15$}

- CKD was when plasma creatinine was $>190 \mu \mathrm{mol} / \mathrm{L}$ or GFR $<60 \mathrm{~mL} / \mathrm{min} / 1.73 \mathrm{~m}^{2}$ or progression of CKD when the patient had worsening of these values compared to the preoperative period.

- ESRD was when the patient has been put on an ESRD program for hemodialysis or renal transplantation or had died of uremia. 


\section{Independent variables 7,9}

- Age at presentation and intervention were considered in months when the patient had been diagnosed with PUVs and consequently at what age the patient received intervention, respectively.

- Deranged renal function test was when the creatinine level was elevated above the normal level according to age.

- Presence of VUR: backflow of urine from bladder to kidney

- Type of procedure: endoscopic ablation and temporarily vesicostomy

- Time delay between development of vesicostomy and PUV ablation

- Nadir serum creatinine: lowest creatinine attained in the year after valve ablation

- Preoperative and postoperative proteinuria: positive protein on urinalysis pre- and postablation, respectively

- Preoperative and postoperative hypertension: blood pressure elevated above 95th percentile for age in pre- and postoperative period, respectively

- Diurnal urinary continence was defined as being totally dry during the day and night.

\section{Results}

A total of 139 patients were ablated following PUV diagnosis between 2015 to 2019. In sum, 92 cards were retrieved and the other 47 had been lost. From the 92 charts found, only 70 had properly documented variables, and thus only these were analyzed. Findings were then documented in the preformed questionnaires and analyzed using SPSS 25.

It was found that around $45.7 \%$ of patients had presented with obstructive symptoms aged $>2$ years, $42.9 \%$ presented aged 1 month to 2 years, only $11.4 \%$ were diagnosed at $<1$ month of age, and none was diagnosed antenatally (Figure 1).

Clinical presentation showed that around $80.4 \%$ presented with difficulty of urination, and recurrent urinary tract infection (UTI) was seen in 4.4\%. Around 50\% had had no documented blood-pressure measurement preoperatively, but for the rest, $31.5 \%$ had had preoperative hypertension. Preoperative proteinuria was present in $47.8 \%$ of patients and absent in $29.3 \%$, but was undocumented in around $22.8 \%$.

Almost $97 \%$ of patients had preoperative creatinine documentation, of which $50 \%$ had normal creatinine levels for their age and around 46.7\% had elevated creatinine. Preoperative ultrasound had been done for all patients and showed that severe bilateral hydroureteronephrosis was seen in $64.8 \%$, while $24 \%$ and $11 \%$ had moderate and mild hydroureteronephrosis, respectively. On ultrasound, from those with documented bladder changes, $68.4 \%$ had significant bladder-wall hypertrophy with trabeculations. Preoperative VCUG had been done in $87 \%$ of patients, of which $60 \%$ had no upper urinary tract abnormality, $23.8 \%$ had unilateral VUR, and $16.3 \%$ bilateral VUR. High-grade VUR had been documented in $58.1 \%$, low-grade VUR 25.8\%, and around 16.1\% had no documentation of VUR grade.

트 age at presentation 트 age at intervention

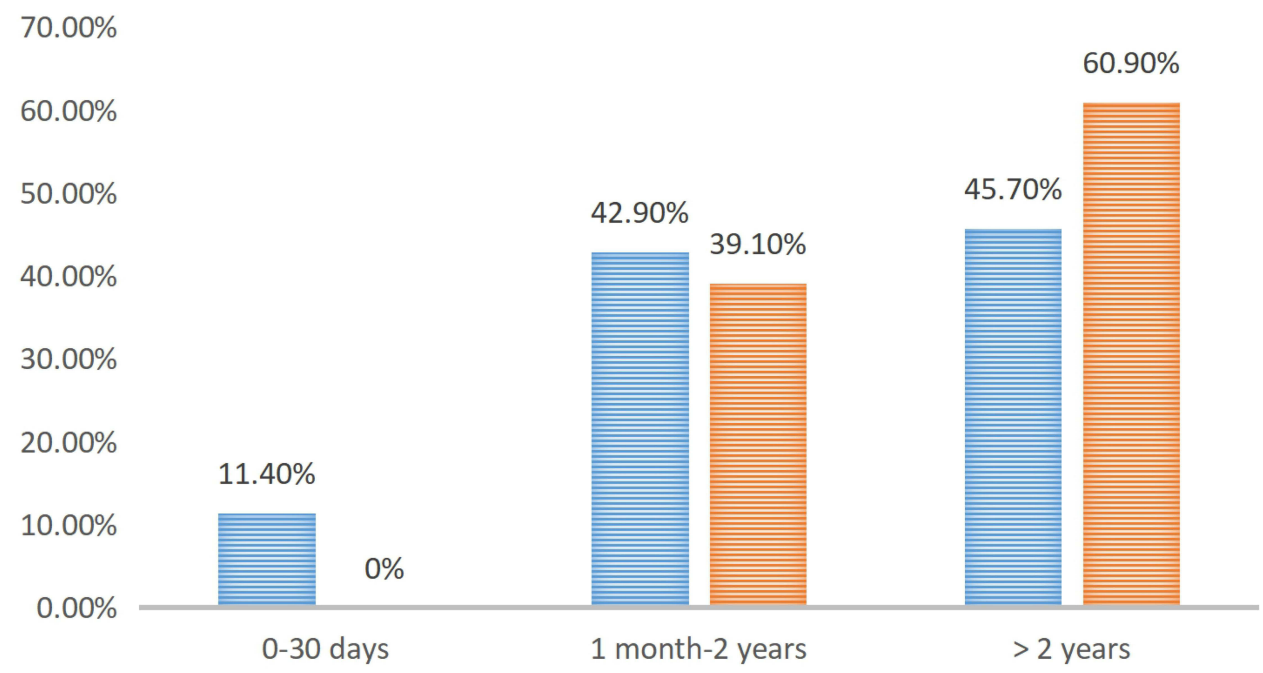

Figure I Differences between age at diagnosis and intervention. 
Table I Frequency of Different Stages of CKD Postablation

\begin{tabular}{|l|l|}
\hline CKD Stage & Postoperative CKD \\
\hline 1 & $7.1 \%$ \\
2 & $15.7 \%$ \\
3 & $18.6 \%$ \\
4 & $8.6 \%$ \\
5 & $2.9 \%$ \\
\hline
\end{tabular}

Preoperative CKD was present in $36.3 \%$ of patients, of which $38.5 \%$ had stage $3 \mathrm{CKD}, 15.4 \%$ stage 4 , and $19.2 \%$ stage 1 (Table 1).

Postoperatively, around $60.9 \%$ of patients had been ablated aged $>2$ years and $39.1 \%$ were aged 1 month to 2 years. Around $65.2 \%$ of patients had had ablation done primarily, while $34.8 \%$ had vesicostomy prior to ablation. Following vesicostomy, around $55.2 \%$ had their ablation done after 1 year vs $44.8 \%$ in $<1$ year (Figure 2 ).

The results showed poor follow-up of patients, with $38.6 \%$ being followed for $<1$ year and around $61.4 \%$ on follow-up at 3 years postoperatively. Since most patients were lost to follow-up at 5 years $(75 \%)$, it was considered difficult to reach a conclusion so, the analysis was changed from 5 years' follow-up to 3 years' follow-up.

Diurnal incontinence was also assessed for those aged 5 years, and it was seen that around 69\% had either no documentation or had the vesicostomy still in place, but around $20 \%$ had diurnal incontinence. Since most patients had no documentation, this variable was not analyzed as one of the risk factors of CKD.
Postoperatively, blood pressure had been documented in around $63.7 \%$ of the charts, of which around $38.5 \%$ had postoperative hypertension and $25.3 \%$ had no hypertension. Postoperative proteinuria was seen in $27.5 \%$ of cases vs $29.7 \%$ with no proteinuria.

The lowest serum creatinine at 1 -year postablation was taken for analysis and a cutoff point of $0.8 \mathrm{mg} / \mathrm{dL}$ was used. Around $36.3 \%$ of patients had low creatinine, $34.1 \%$ had higher creatinine than the cutoff point, and around $29.7 \%$ had no documented nadir serum creatinine.

Postoperatively, recurrent UTI was seen in $27.6 \%$ and around 57\% had no UTI. Also, 58.7\% had had an abdominal ultrasound done postoperatively, of which $41.5 \%$ showed improvement from their preoperative ultrasound, $26.4 \%$ had worsening, and $32.1 \%$ had similar findings. Since most of these patients had abdominal ultrasounds taken at different times, the 1-year ultrasound was used for analyzsis. Postoperative VCUG had been ordered for those patients with obstructive symptoms following ablation (38.6\%), and it was seen that $14.8 \%$ had normal VCUG findings, $18.5 \%$ had membranous urethral stricture, and $25.9 \%$ had documented remnant valve, of which around $60 \%$ had repeat ablations.

It was seen that $71.9 \%$ of patients had no documented parameters to consider or exclude bladder dysfunction Since most of the patients had no documentation, this variable was difficult to analyze as a prognostic factor for CKD and was thus excluded from the study.

Three-year renal outcomes showed that there were 52.9\% of patients with postoperative CKD, while $47.1 \%$ had not

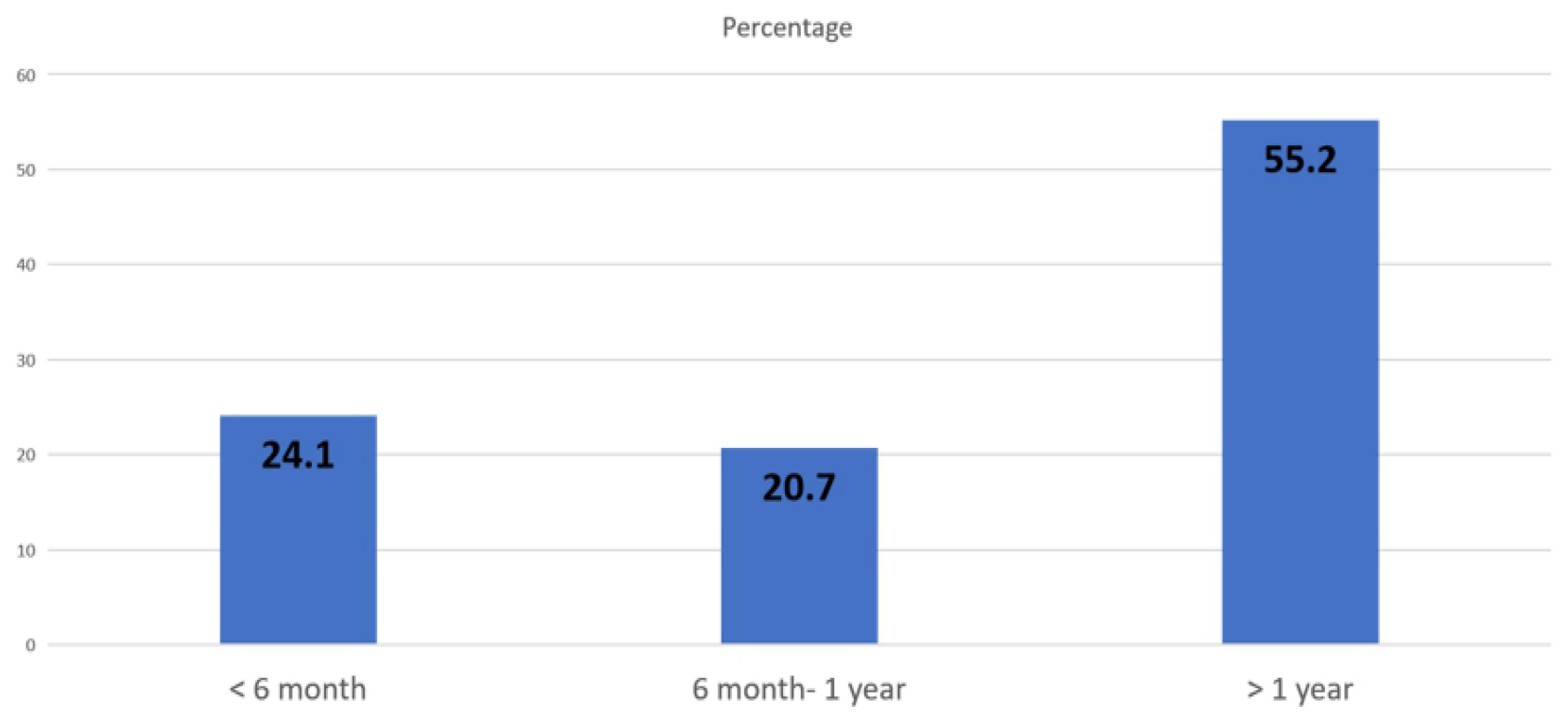

Figure 2 Time interval between vesicostomy and ablation. 
developed CKD (Table 1). The study's main objective was to study the short- and long-term renal outcomes of PUVs, but since most patients were lost to follow up, 3-year CKD was used as a dependent variable and analyzed.

Cross-tabulation with $\chi^{2}$ was then done between the independent and dependent variables, and it was seen that there was a significant correlation between development of preoperative proteinuria and CKD. Postoperative hypertension and proteinuria also correlated with development of CKD. An association was also found between elevated nadir serum creatinine and CKD (Table 2).

Age at presentation and intervention was then analyzed for future development of CKD and no correlation was found, while those patients that had had vesicostomy and ablation done consecutively were analyzed for future development of CKD, and it was found that those with delayed ablation after vesicostomy showed statistical significance. Otherwise, no other variable was found to be statistically significant.

Significant values were then analyzed with logistic regression, and remained significant for three variable: elevated nadir serum creatinine, postoperative proteinuria, and delay between development of vesicostomy and ablation. When evaluating ORs, it was seen that all had risks of 6.9-, 14.7-, and 2.8-fold for development of CKD, respectively. These three variables were thus considered as independent risk factors for development of CKD (Table 3).

\section{Discussion}

In developing countries, there is scanty reference to PUV and diagnosis is often delayed due to lack of awareness and adequate facilities for investigation. ${ }^{9}$ In this study there were no patients with antenatal diagnosis, and most had been diagnosed aged $>2$ years.

In Nigeria, PUV is considered to be the most common cause of lower urinary tract anomaly, found in $15 \%$ of cases; ${ }^{9}$ however, diagnosis is delayed in the neonatal period due to

Table 2 Statistically Significant Variables (Pearson's $\chi^{2}$ )

\begin{tabular}{|l|l|}
\hline & $P$ \\
\hline Preoperative hypertension & 0.06 \\
Preoperative creatinine & 0.08 \\
Delay between development of vesicostomy and ablation & 0.038 \\
Preoperative proteinuria & 0 \\
Postoperative proteinuria & 0 \\
Postoperative hypertension & 0 \\
Nadir serum creatinine & 0.001 \\
\hline
\end{tabular}

Table 3 Independent Risk Factors Related to Postoperative CKD on Multivariate Statistical Analysis

\begin{tabular}{|l|l|l|l|}
\hline & $P$ & CI & OR \\
\hline Elevated nadir serum creatinine & 0.001 & $2.228-21.454$ & 6.914 \\
Postoperative proteinuria & 0.000 & $3.410-64.886$ & 14.87 \\
$\begin{array}{l}\text { Delay between development of } \\
\text { vesicostomy and ablation }\end{array}$ & 0.038 & $1.061-7.581$ & 2.836 \\
Preoperative proteinuria & 0 & $0.116-2.128$ & \\
Postoperative hypertension & 0 & $0.011-1.202$ & \\
\hline
\end{tabular}

nonurological presentation, and thus mortality (24\%) is high. ${ }^{9}$ In this study, there was no antenatal diagnosis and around $45.7 \%$ had been diagnosed aged $>2$ years, and thus $>60 \%$ also received intervention aged $>2$ years.

This study showed that $52.9 \%$ of patients had developed CKD within 3 years of follow-up, and ESRD was seen in $2.9 \%$. A study from Rome followed 24 patients for 12 years, and $20.8 \%$ of patients had ESRD and $54.3 \%$ CKD. ${ }^{13}$

Risk factors found to be significant for the development of postoperative CKD were preoperative and postoperative proteinuria, postoperative hypertension, high nadir serum creatinine, and delay between development of vesicostomy and ablation. Independent risk factors were elevated nadir serum creatinine, postoperative proteinuria, and delay between development of vesicostomy and ablation, whereas in the Rome study bilateral VUR and postoperative hypertension were associated with CKD. ${ }^{11}$

In Finland, 193 patients were followed for a median of 31 years, and it was found that early presentation, high serum creatinine, bilateral VUR, and recurrent UTI were associated with ESRD (22.8\%). ${ }^{12}$ Another study done in Finland also shows that bilateral VUR have decreased overall kidney function. ${ }^{14}$ Though our ESRD (2.9\%) was low compared to the study, our follow-up was only 3 years.

In the UK, age at presentation is seen as a risk factor, where $41 \%$ of patients aged $<1$ year vs $15 \%$ of patients older than 1 year show poor long-term renal outcome, but in this study this was found to have no statistical significance. $^{10}$

Among the few studies in Africa, one in Egypt followed PUV patients for 4 years and found no correlation between age at presentation and long-term renal outcome. ${ }^{7}$ The same study found a correlation between elevated nadir serum creatinine and bilateral VUR for poor long-term outcomes. ${ }^{7}$

A study from Nigeria followed 23 patients that had primarily undergone vesicostomy, and concluded it to be safe in respect to long-term outcome and that it should be considered as an initial management of PUVs in a setup 
where the proper instrumentation is not available. ${ }^{19}$ While a study from Washington showed best result when ablation was done in the first month of life and severe renal insufficiency tends to progress even with upper tract diversion. ${ }^{17}$

Delay between development of vesicostomy and ablation in our study was found to have significance for CKD, and on logistic regression it was shown to be an independent risk factor, with 2.8 times the risk of developing CKD.

In Ethiopia, the chance of diagnosing PUV in the prenatal period is low, because there are few mothers that attend proper antenatal care and undergo ultrasound examination. Also, there is a lack of sound knowledge in diagnosing and treating these patients early in different setups. As such, these patients come to the hospital after developing symptoms during infancy and toddlerhood. In a Nigerian study, it was concluded that increased awareness of the condition and its varied clinical presentation will help in early recognition and improvement in prognosis. ${ }^{9}$

Following diagnosis, intervention was done at only one center that had a high patient load, thus causing delays. There was also only one poorly functioning, out-of-date machine for all these patients, and thus patients in this setup might have needed more than one intervention. Due to all these reasons, a PUV patient with alreadycompromised kidney would have deteriorated. Our study showed that obstructive symptoms following ablation developed in $38.6 \%$ for whom VCUG had been done, of which $25.9 \%$ had remnant valves. A study from Turkey followed 101 patients following ablation for 1 year and found around $7.9 \%$ had remnant valves. ${ }^{20}$

\section{Conclusion}

We found a high rate of postoperative CKD (52.9\%) comparable to other studies done worldwide. It was also noticed in this study that preoperative and postoperative proteinuria, postoperative hypertension, high nadir serum creatinine, and delay between development of vesicostomy and ablation were associated with development of long-term renal complications, of which elevated nadir serum creatinine, postoperative proteinuria, and delay between development of vesicostomy and ablation were all independent risk factors, unlike other findings seen in low- and middle-income countries.

\section{Limitations and Recommendations}

This was a retrospective study, so some of the charts were lost and of those enrolled, some variables had not been properly documented and thus could not be analyzed. Most patients were lost from follow-up at around 5 years, and thus it was difficult to assess long-term renal outcomes.

Based on this study, it is our recommendation that all correctable risk factors for the development of CKD should be sought and dealt with early.

All patients need extended follow-up at both both urology and nephrology clinics via phone or physically with dedicated clinic days given to them.

Finally, it is our recommendation that a large prospective study be done to estimate the incidence of ESRD in our setup following PUV ablation.

\section{Acknowledgment}

We would like to thank the Pediatric Surgery Unit of the Department of Surgery, Tikur Anbessa Specialized Hospital, for encouraging this research.

\section{Disclosure}

The authors report no conflicts of interest in this work.

\section{References}

1. Wein AJ, Kavoussi LR, Partin AW, Peters CA. Campbell Walsh Urology. 11th ed.; Elsevier, 2016:4318-4321.

2. Docimo SG, Canning DA, Khoury AE. The Kelalis-King-Belman Textbook of Clinical Pediatric Urology. 5th ed. Informa Health Care. 2007:1059-1060.

3. Gearhart JP, Rink RC. Pediatrics Urology. 2nd ed. Saunders imprint of Elsevier; 2010:437-438.

4. Tadesse A, Gossaye A, Derbew M. Pediatrics surgical admissions and procedures in Tikur Anbessa specialized hospital, Ethiopia: a five-year retrospective study. Ethiop Med J. 2019;57(2).

5. Holcomb GW III, Patrick Murphy J, St Peter SD. Holcomb and Ashcraft's Pediatric Surgery. 7th ed.; 2019:884-885.

6. Karmarkar SJ. Long term results of posterior urethral valves: a review. Pediatr Surg Int. 2001;17:8-10. doi:10.1007/s00383000 0481

7. Sarhan OM, El-Ghoneimi AA, Helmy TE, Dawaba MS, Ghali AM, Ibrahiem E-HI. Posterior urethral valves: multivariate analysis of factors affecting the final renal outcome. $J$ Urol. 2011;18 5:2491-2496. doi:10.1016/j.juro.2011.01.023

8. Coran AG, Scott Adzick N, Krummel TM, Jean-Martin Laberge RC, Shamberger AA. Caldamone, Coran Pediatrics Surgery. 7th ed.; 2012:1556-1557.

9. Garg SK, Abdurrahman MB, Momoh JT, Hargreaves HM, Narayana P, Lawrie JH. Congenital posterior urethral valves: problems of management in countries with limited facilities. Ann Trop Paediatr. 1983;3:201-205. doi:10.1080/02724936.1983.11748295

10. Parkhouse HF, Barratt TM, Dillon MJ, et al. Long-term outcome of boys with posterior urethral valves. Br J Urol. 1988;62(1):59-62. doi:10.1111/j.1464-410x.1988.tb04267.x

11. Tirlea S, Lonescu S. Posterior urethral valves-diagnosis and primary treatment considerations. Chirurgia (Bucur). 2012;107(2):218-225. PMID: 22712352.

12. Heikkilä J, Holmberg C, Kyllönen L, Rintala R, Taskinen S. Longterm risk of end stage renal disease in patients with posterior urethral valves. J Urol. 2011;186:2392-2396. doi:10.1016/j.juro.2011.07.109 
13. Caione P, Nappo SG. Posterior urethral valves: long-term outcome. Pediatr Surg Int. 2011;27:1027-1035. doi:10.1007/s00383-0112946-9

14. Dinneen MD, Duffy PG. Posterior urethral valves. Br J Urol. 1996;78 (2):275-281. doi:10.1046/j.1464-410x.1996.10324.x

15. Heikkilä J, Rintala R, Taskinen S. Vesicoureteral reflux in conjunction with posterior urethral valves. J Urol. 2009;182(4):1555-1560. doi:10.1016/j.juro.2009.06.057

16. Johnston JH, Kulatila AE. The sequelae of posterior urethral valve. $B r J$ Urol. 1971;43(6):743. doi:10.1111/j.1464-410x.1971. tb12098.x

17. Close CE, Carr MC, Burns MW, Mitchell ME. Lower urinary tract changes after early valve ablation in neonates and infants: is early diversion warranted? J Urol. 1997;157:984-988. PMID: 9072631. doi:10.1016/S0022-5347(01)65125-5
18. Farhat W, Mclorie G, Capolicchio G, Khoury A, Bagli D, Mergueria PA. Outcomes of primary valve ablation versus urinary tract diversion in patients with posterior urethral valves. Urology. 2000;56:653-657. doi:10.1016/S0090-4295(00)00784-6

19. Lukong CS, Ameh EA, Mshelbwala PM, et al. Role of vesicostomy in the management of posterior urethral valve in Sub-Saharan Africa. J Pediatr Urol. 2014;10:62e66. doi:10.1016/j.jpurol.2013.06.004

20. Oktar T, Salabas E, Acar O, et al. Residual valve and stricture after posterior urethral valve ablation: how to evaluate? J Pediatr Urol. 2013;9:184-187. doi:10.1016/j.jpurol.2012.01.016

\section{Publish your work in this journal}

Research and Reports in Urology is an international, peer-reviewed, open access journal publishing original research, reports, editorials, reviews and commentaries on all aspects of adult and pediatric urology in the clinic and laboratory including the following topics: Pathology, pathophysiology of urological disease; Investigation and treatment of urological disease; Pharmacology of drugs used for the treatment of urological disease. The manuscript management system is completely online and includes a very quick and fair peer-review system, which is all easy to use. Visit http://www.dovepress.com/ testimonials.php to read real quotes from published authors. 